\title{
Avaliação da Qualidade de uma Comunicação VoIP em uma Rede Ethernet LAN e WLAN
}

\author{
Felipe Mariano da Silva e Demóstenes Zegarra Rodríguez
}

\begin{abstract}
Resumo-Este trabalho tem como objetivo comparar a qualidade de uma comunicação VoIP em uma rede com fio (Ethernet LAN) e em uma rede sem fio (WLAN), e avaliar o grau de degradação que um sinal interferente (Bluetooth) pode causar em uma comunicação VoIP. Esta comparação será efetuada individualmente para os codificadores de voz G.711, G723.1 e iLBC, tendo como parâmetros de medida o índice MOS, determinado pela Recomendação ITU-T P.862, com a utilização dos parâmetros de rede: perda de pacotes e atrasos. Os testes efetuados neste trabalho foram realizados em um cenário de emulação.
\end{abstract}

Palavras-Chave-VoIP, Codec, P.862, MOS, Bluetooth.

Abstract-This work aims to compare the quality of a communication VoIP into a wired network (Ethernet LAN) and a wireless network (WLAN), and it measures the degree of degradation that a signal interference (Bluetooth) can cause in a communication VoIP. This comparison will be made individually for the voice codecs G.711, iLBC and G723.1, with the parameters of measure the index MOS, given by Recommendation ITU-T P.862, using the parameters of network : packets loss and delays. The tests performed in this work were carried out in a scenario of emulation.

Keywords - VoIP, Codec, P.862, MOS, Bluetooth.

\section{INTRODUÇ̃̃O}

Nos últimos anos as comunicações sem fios (WLAN) vêm tendo uma maior importância que as redes com fio (Ethernet LAN) devido ao crescimento dos dispositivos sem fio e por sua facilidade de implantação e configuração.

Quando um sinal é transmitido no ar ocorre uma degradação por diferentes fatores externos como: interferência de outros sinais, condições climáticas, obstáculos (paredes) existentes entre o transmissor e o receptor, mobilidade dos usuários finais entre outros. Portanto, estudar como estes parâmetros se relacionam é muito relevante.

Neste trabalho é apresentada a emulação de um cenário Ethernet LAN e WLAN com a presença de ligações de chamada de voz sobre uma rede IP (VoIP), sendo que todas as ferramentas utilizadas são open source, resultando em um cenário de fácil reprodução para futuras pesquisas.

Os primeiros testes foram realizados em um cenário de rede Ethernet LAN e foram empregados os codificadores de voz: G.711 [1], G.723.1 [2] e iLBC [3]. Estes codificadores estão presentes em trabalhos de pesquisa atuais.

Para a determinação do valor do índice MOS (Mean Opinion Score) é utilizada a Recomendação ITU-T P.862 [4] geralmente conhecida como PESQ (Perceptual Evaluation of

Felipe Mariano da Silva e Demóstenes Zegarra Rodríguez, Laboratório de Telecomunicações do Instituto Nokia de Tecnologia, Manaus, Brasil, E-mails: silvafelipe.A@indt.org.br, demostenes.rodriguez@indt.org.br.
Speech Quality), em termos gerais esta recomendação descreve um método objetivo de avaliação da qualidade subjetiva de codificadores. Na recomendação ITU-T P.800 [5] se apresenta a escala da pontuação MOS que vai desde 1, para comunicações inteligíveis, até 5 para comunicações de ótima qualidade.

Verificou-se a não existência de sinais que pudessem interferir ou causar alguma degradação na comunicação. Em seguida, foi introduzido um sinal Bluetooth para avaliar como este sinal interferente degradaria a qualidade do sinal de voz transmitida.

Os parâmetros que foram avaliados, além do índice $M O S$, foram: probabilidade de perda de pacotes (PLR), atrasos utilizando um analisador de rede e a análise de interferência de sinais utilizando o analisador de espectro.

\section{Cenário de Emulação}

Para a realização dos testes será implementado um cenário emulado de comunicação VoIP sobre uma rede WLAN (Wireless Local Area Network). A seguir são descritas as ferramentas que serão empregadas neste trabalho.

- Servidor para áudio interativo: utilizou-se o softphone freeware Myphone [6].

- Analisador de Rede: utilizou-se o software freeware Wireshark 0.99.7 System [7].

- Software de gravação de som: foi utilizada a versão freeware do software VRS Recording System [8].

A Figura 1 apresenta o cenário emulado de uma rede cabeada e a Figura 2 apresenta a rede WLAN.

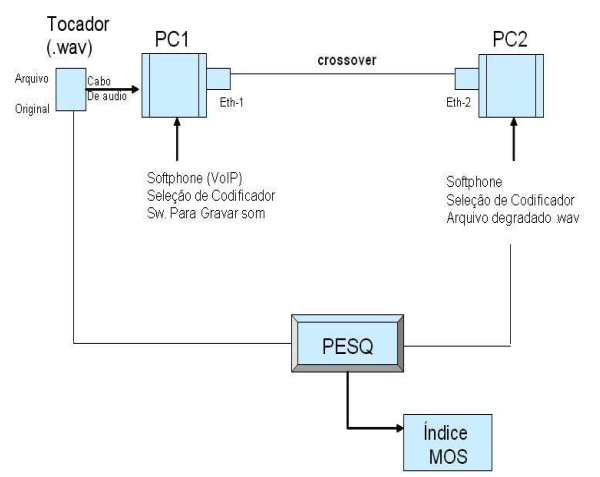

Fig. 1. Cenário de testes com rede Ethernet LAN.

Antes da realização dos testes do cenário da Figura 2 verificou-se no analisador de espectro EXA Signal Analyzer [9] a não existência de sinais que pudessem degradar o sinal transmitido, como é observado na Figura 3. 


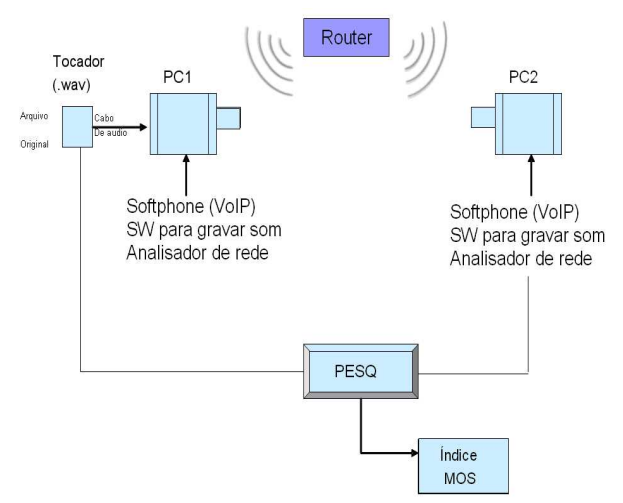

Fig. 2. Cenário de testes com rede WLAN.

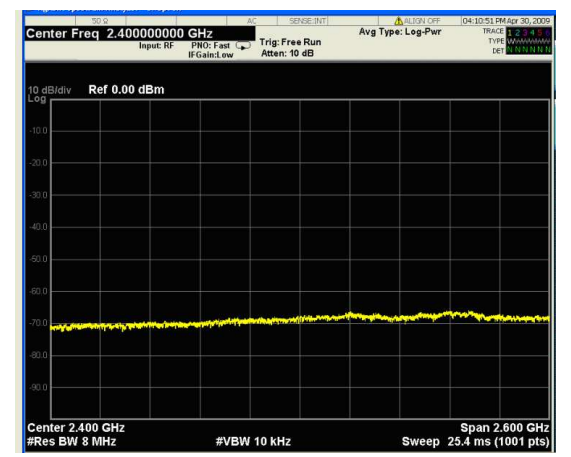

Fig. 3. Espectro com frequiência central de $2.4 \mathrm{GHz}$.

\section{A. Metodologia de Teste}

- Se inicia uma comunicação entre os computadores PC1 e PC2, os softphones fazem a seleção do codificador de voz.

- O reprodutor de áudio transmite o arquivo (arq-orig.wav) ao computador PC1, onde o som é gravado (arqorig2.wav), como a chamada já está ativa, a voz é codificada e transmitida para o computador PC2.

- Enquanto os dados são transmitidos o programa Wireshark, no PC1 e no PC2, salva toda a informação da rede.

- No PC2, o áudio recebido é gravado em um arquivo (arqdeg.wav). Este último arquivo e o arquivo arq-orig2.wav são comparados mediante o programa $P E S Q$, que é executado no $\mathrm{PC} 2$, tendo como resultado uma pontuação MOS.

\section{RESUltados}

A Tabela I apresenta os valores obtidos para ambos cenários de rede que foram testados, considerando os diferentes codificadores de voz avaliados. Os testes do cenário de rede WLAN foram realizados a uma distancia de 8 metros entre o emissor e receptor sem nenhum obstáculo e sinal interferente.

\section{A. Interferência com sinal Bluetooth}

Realizaram-se testes no cenário de rede WLAN adicionando um sinal Bluetooth com o propósito de medir o grau de degradação do sinal de voz transmitida. Os resultados obtidos são apresentados na Tabela II.
TABELA I

VALORES MOS E PLR (\%) PARA CENÁRIOS DE REDE Ethernet LAN E WLAN

\begin{tabular}{|c||c|c|c|c|}
\hline \multicolumn{1}{|c||}{} & \multicolumn{2}{c|}{$\begin{array}{c}\text { Rede } \\
\text { Ethernet LAN }\end{array}$} & \multicolumn{2}{c|}{ Rede WLAN } \\
\hline \hline Codificador & MOS & PLR & MOS & PLR \\
\hline G.711(64 kbit/s) & 4,09 & 0 & 4,022 & 5 \\
\hline G.723.1(5,3 kbit/s) & 3,524 & 0 & 3,426 & 7 \\
\hline iLBC $(15,2 \mathrm{kbit} / \mathrm{s})$ & 3,793 & 0 & 3,774 & 4 \\
\hline
\end{tabular}

TABELA II

VALORES MOS E PLR (\%) PARA CENÁRIOS COM E SEM INTERFERÊNCIA

\begin{tabular}{|c||c|c|c|c|}
\hline \multicolumn{1}{|c||}{} & \multicolumn{2}{|c|}{$\begin{array}{c}\text { Rede WLAN } \\
\text { sem sinal } \\
\text { interferente }\end{array}$} & \multicolumn{2}{c|}{$\begin{array}{c}\text { Rede WLAN } \\
\text { com sinal } \\
\text { Bluetooth }\end{array}$} \\
\hline \hline Codificador & MOS & PLR & MOS & PLR \\
\hline G.711(64 kbit/s) & 4,022 & 5 & 3,902 & 11 \\
\hline G.723.1(5,3 kbit/s) & 3,426 & 7 & 3,236 & 13 \\
\hline iLBC $(15,2 \mathrm{kbit} / \mathrm{s})$ & 3,774 & 4 & 3,618 & 10 \\
\hline
\end{tabular}

\section{CONCLUSÕES}

Dos resultados obtidos pode-se observar que uma comunicação WLAN tem uma qualidade menor que uma comunicação Ethernet LAN, isto ocorre devidoa aos diferentes tipos de degradações no canal rádio-frequência (RF), tais como: fading e shadowing.

Em trabalhos futuros serão avaliados os diferentes modelos de fading, utilizando um emulador de canal RF para determinar como cada um destes modelos afeta a qualidade de uma comunicação de voz em uma rede WLAN.

Também pode-se concluir que a degradação da qualidade em um ambiente WLAN depende também do tipo de codificador utilizado, pois como foi observado o codificador iLBC teve uma melhor resposta em relação aos outros dois codificadores de voz avaliados (G.711 e G723.1).

\section{AGRADECIMENTOS}

Os autores agradecem ao Instituto Nokia de Tecnologia pela motivação à pesquisa e por disponibilizar os equipamentos necessários para a implementação dos cenários de teste.

\section{REFERÊNCIAS}

[1] ITU-T Rec. G.711, General Aspects of Digital Transmission Systems Terminal Equipments - Pulse Code Modulation (PCM) of Voice Frequencies. 1972. http://www.itu.int/rec/T-REC-G.711/en.

[2] ITU-T Rec. G.723.1, General Aspects of Digital Transmission Systems - Dual Rate Speech Coder for Multimedia Communications Transmitting at 5.3 and $6.3 \mathrm{kbit} / \mathrm{s}$. May. 2006. http://www.itu.int/rec/T-RECG.723.1/en.

[3] iLBC - IETF RFC 3951, Internet Low Bit Codec Rate. Dec. 2004. Disponível em http://www.ietf.org/rfc/rfc3951.txt.

[4] ITU-T Rec. P.862, Perceptual evaluation of speech quality (PESQ): An objective method for end-to-end speech quality assessment of narrowband telephone networks and speech codecs. http://www.itu.int/rec/TREC-P.862/en.

[5] ITU-T Rec. P.800, Methods for subjective determination of transmission quality. Aug. 1996. Disponível em: www.itu.int/rec/T-REC-P.800/en.

[6] Softphone Myphone, http://myphone.sourceforge.net/.

[7] Wireshark, http://www.wireshark.org/download.html.

[8] VRS Recording System, Disponível em: www.nch.com.au/vrs/index.html.

[9] EXA Signal Analyzer N9010A, Agilent Technologies, www.agilent.com/find/exa-manuals. 